\title{
Homogeneity of Bilayer Graphene
}

\author{
Frank Freitag ${ }^{\mathrm{a}}$, Markus Weiss ${ }^{\mathrm{a}, *}$, Romain Maurand ${ }^{\mathrm{a}}$, Jelena Trbovic ${ }^{\mathrm{a}}$, \\ Christian Schönenberger ${ }^{\mathrm{a}}$ \\ ${ }^{a}$ Department of Physics, University of Basel, Klingelbergstr. 82, CH-4056 Basel, \\ Switzerland
}

\begin{abstract}
We present non-linear transport measurements on suspended, current annealed bilayer graphene devices. Using a multi-terminal geometry we demonstrate that devices tend to be inhomogeneous and host two different electronic phases next to each other. Both of these phases show gap-like features of different magnitude in non-linear transport at low charge carrier densities, as already observed in previous studies. Here, we investigate the magnetic field dependence and find that both features grow with increasing field, the smaller one with $0.6 \mathrm{meV} / \mathrm{T}$, the larger one with a 5-10 times higher field dependence. We attribute the larger of the two gaps to an interaction induced broken symmetry state and the smaller one to localization in the more disordered parts of the device.
\end{abstract}

Keywords: A. Bilayer graphene; A. Multiterminal device; D. Broken symmetry groundstate; E. Nonlinear transport.

\section{Introduction}

The isolation of monolayer graphene [1] has started a new research field on two-dimensional carbon based electronic systems. Whereas the interest in monolayer graphene mainly stems from its unusual pseudo-relativistic electron dispersion, bilayer graphene promises to be interesting because of its strongly interacting charge carriers. Weak screening in suspended samples and small carrier densities at the charge neutrality point lead to large values for the interaction parameter $\mathrm{r}_{S}$ about one order of magnitude larger than in conventional 2DEGs of comparable carrier density [2]. For interaction effects to be observable in electronic transport experiments, bilayer graphene has to be very clean to achieve low charge carrier densities. To reach this regime, the normally used $\mathrm{SiO}_{2}$ substrate has to be removed by underetching, leaving the bilayer freely suspended between the contacts [3, 4]. The suspension allows to current anneal the bilayer graphene flake by Joule heating induced by passing a large current

\footnotetext{
* Corresponding author

Email address: Markus.Weiss@unibas.ch (Markus Weiss)
} 
through it. During this heating cycle adsorbates may (at least partially) evaporate.

The exact nature of the electronic groundstate of bilayer graphene has been investigated theoretically for several years [5-15]. The fourfold degeneracy that stems from the spin and valley degrees of freedom of the charge carriers will be broken by interactions, and new groundstates with partly or totally lifted degeneracies are expected to occur. Interaction induced broken-symmetry states that are being discussed as possible candidates for the groundstate of bilayer graphene include the quantum anomalous Hall insulator (QAH) 11, 12], the quantum spin Hall insulator (QSH) 12], the nematic phase 16, 17], the layer antiferromagnetic phase (LAF) [12, 14, 18], and possibly more. Most, but not all of these states show a gap in the excitation spectrum around $E_{F}=0$, leading to a vanishing conductivity around the charge neutrality point (CNP).

We can probe the electronic properties of the graphene bilayer as a function of temperature, charge carrier density and magnetic field by measuring the differential conductance $d I / d V=G_{d}$ as a function of a bias voltage $V_{s d}$. We investigate low conducting and insulating samples with similar features as in a previous study, that exhibit two distinct gap-like features in $G_{d}\left(V_{s d}\right)[19]$, and that mainly differ in the minimum conductance at $V_{s d}=0$. Here, we further investigate these kinds of samples by looking at the magnetic field dependence of $G_{d}\left(V_{s d}\right)$ [20]. Our results suggest that the samples are inhomogeneous after current annealing and are composed of two different electronic phases. A possible reason for this is that the process of current annealing does not clean the samples in a uniform way, but creates almost disorder free areas in the center of the samples and leaves some disorder at the edges, which leads to a coexistence of electronic phases next to each other.

\section{Material and methods}

Bilayer graphene was deposited onto a $\mathrm{p}^{+}$-doped silicon wafer covered with $300 \mathrm{~nm}$ silicon dioxide by micromechanical cleavage of natural graphite. After deposition, a thin $(5 \mathrm{~nm})$ film of aluminium was evaporated over the whole wafer. After exposure to air, this film oxidised and transformed to $\mathrm{Al}_{2} \mathrm{O}_{3}$ almost completely. Using standard electron-beam lithography, a PMMA etchmask was then structured for shaping the graphene flake using an $\mathrm{Ar} / \mathrm{O}_{2}$ plasma, and in a second lithography step, the electrode structures were defined. After each resist development, the exposed part of the oxidised aluminium film was removed in a $25 \%$ tetramethylammonium hydroxide (TMAH) solution, thereby eliminating any organic residues left on the graphene. The electrode structures $(3 \mathrm{~nm} \mathrm{Cr}$ and $70 \mathrm{~nm} \mathrm{Au}$ ) were e-gun evaporated, and subsequently the graphene devices were annealed in high vacuum at $10^{-7} \mathrm{mbar}$ and $200^{\circ} \mathrm{C}$ for several hours. The silicon dioxide was then partly removed in $2 \%$ buffered hydrofluoric acid, leaving the graphene suspended $160 \mathrm{~nm}$ above the substrate. The remaining silicon wafer acted as a gate with a voltage $V_{g}$ applied. The devices were mounted into a ${ }^{3} \mathrm{He}$ cryostat in vacuum and subsequently current annealed at $T=1.5 \mathrm{~K}$ with typically $0.4 \mathrm{~mA}$ per $\mu \mathrm{m}$ of flake width [19]. For the present study, we fabricated 
devices with a four-terminal Hall cross geometry (insets of fig. 1); the longer axis was about $3 \mu \mathrm{m}$ long, the shorter $1.5 \mu \mathrm{m}$ and the width of each individual arm was $1 \mu \mathrm{m}$. Current annealing was done on pairs of contacts with the other two contacts floating. The annealing process was considered to be successful if a pronounced dip in $G$ measured as a function of the gate-voltage $V_{g}$ appeared in the vicinity of $V_{g}=0$, i.e. at low charge carrier density of at least one contact pair combination.

\section{Results}
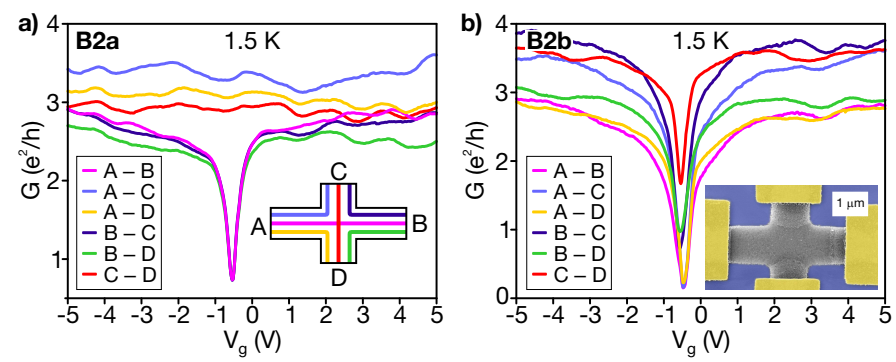

Figure 1: (a) Two-terminal conductance $G$ of a Hall-cross device as function of the gate voltage $V_{g}$ at $1.5 \mathrm{~K}$ in device $\mathrm{B} 2 \mathrm{a}$. The possible configuration are shown in the inset. Measurements involving contact $B$ show a pronounced dip in $G$ at $V_{g} \approx-0.5 \mathrm{~V}$. The remaining contact pairs have a flat gate response. (b) $G$ as a function of $V_{g}$ for all possible combinations of contacts in device B2b at $1.5 \mathrm{~K}$. All curves show a minimum in $G$ at $V_{g} \approx-0.5 \mathrm{~V}$. The inset shows a scanning electron micrograph of the device.

We present data taken on two devices that could successfully be current annealed. Fig. 1 summarises our findings. In the first device, B2a, the current annealing resulted in an inhomogeneous cleaning of the graphene. Fig. 1a) demonstrates that only in two-terminal measurements of $G\left(V_{g}\right)$ that involve contact $\mathrm{B}$ a CNP is visible with a minimum conductance of $0.9 \mathrm{e}^{2} / \mathrm{h}$. For all other contact pairs G only fluctuates around $3 \mathrm{e}^{2} / \mathrm{h}$ as $V_{g}$ is changed and does not display a notable minimum. Further annealing rendered the sample more inhomogeneous, leading to gate characteristics with several CNPs at different $V_{g}$. In contrast, in B2b the current annealing yielded a more homogeneous device. Fig. 1b) shows $G$ as function of $V_{g}$ for B2b. A CNP at $V_{g} \approx-0.5 \mathrm{~V}$ is well developed for all contact pairs. A minimum of $G \approx 0.2 \mathrm{e}^{2} / \mathrm{h}$ at the CNP can be found in $G\left(V_{g}\right)$ measured between contacts A and B. At higher doping, the conductance saturates at $3-4 \mathrm{e}^{2} / \mathrm{h}$.

Performing differential conductance measurements $G_{d}\left(V_{s d}\right)$ reveals further differences between B2a and B2b. In fig. 2 $2 \mathrm{a}$ ), a colour scale plot of $G_{d}$ in $\mathrm{B} 2 \mathrm{a}$ as a function of $V_{g}$ and $V_{s d}$ at $230 \mathrm{mK}$ is shown. The line profile at the $\mathrm{CNP}$ at $V_{g} \approx-0.5 \mathrm{~V}$ exhibits two gap-like features: a smaller one, called $\delta$ in the following, is present up to $\left|V_{s d}\right|=0.6 \mathrm{mV}$ and reduces $G_{d}$ from $1 \mathrm{e}^{2} / \mathrm{h}$ to $0.5 \mathrm{e}^{2} / \mathrm{h}$ at zero $V_{s d}$. The larger feature $\Delta$ is visible up to $\left|V_{s d}\right|=3.5 \mathrm{mV}$ and lowers $G_{d}$ from $3 \mathrm{e}^{2} / \mathrm{h}$ to about $1 \mathrm{e}^{2} / \mathrm{h}$ at zero $V_{s d}$. Moreover, $\Delta$ shows a 

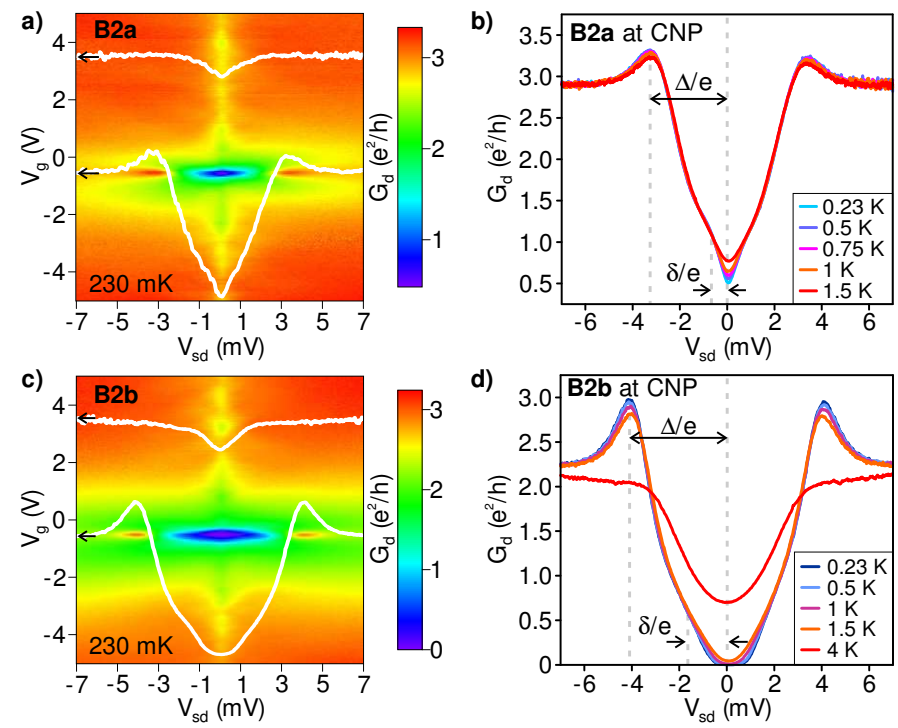

Figure 2: (a) Differential conductance $G_{d}$ as a function of gate voltage $V_{g}$ and bias voltage $V_{s d}$ for device B2a at $230 \mathrm{mK}$. Curves overlaid in white are $G_{d}\left(V_{s d}\right)$-traces taken at gate voltages indicated by the black arrows. Close to the CNP at $V_{g} \approx-0.5 \mathrm{~V} G_{d}$ is suppressed to below $1 \mathrm{e}^{2} / \mathrm{h}$ for small bias voltages. At $\left|V_{s d}\right|=3.5 \mathrm{mV} G_{d}$ reaches a maximum and then saturates around $3 \mathrm{e}^{2} / \mathrm{h}$. Away from the CNP, only a weak dependence on $V_{s d}$ is observable. (b) Temperature dependence of $G_{d}$ as a function of $V_{s d}$ of device B2a at the CNP. Two distinct gap features can be identified. The smaller one, $\delta / e \approx 0.6 \mathrm{mV}$, is reduced when $T$ is raised from $0.23 \mathrm{~K}$ to $1.5 \mathrm{~K}$. The larger gap, $\Delta / e \approx 3.5 \mathrm{mV}$, remains unchanged. (c) $G_{d}$ as a function of $V_{g}$ and $V_{s d}$ for device B2b at $230 \mathrm{mK}$. At the CNP, $V_{g} \approx-0.5 \mathrm{~V}$, and around zero $V_{s d}$ the device is insulating. Changing $V_{g}$ or applying $V_{s d}$ recovers $G_{d} \approx 3 \mathrm{e}^{2} / \mathrm{h}$. (d) Temperature dependence of $G_{d}$ as a function of $V_{s d}$ at the CNP of B2b. The smaller gap, $\delta / e \approx 1.5 \mathrm{mV}$, is reduced when $T$ is increased from $0.23 \mathrm{~K}$ to $1.5 \mathrm{~K}$. The larger gap, $\Delta / e \approx 4 \mathrm{mV}$, is only reduced at $4 \mathrm{~K}$.

BCS-like overshoot in $G_{d}$. Moving $V_{g}$ away from the CNP rapidly suppresses $\Delta$. On the other hand, $\delta$ persists as a small zero bias anomaly even in the metallic regime and is visible in the whole accessible gate voltage range. In fig. 2 $b$ ) the temperature dependence of $G_{d}$ at the CNP of B2a is plotted. The smaller gap feature, $\delta$, is totally suppressed if $T$ increases from $0.23 \mathrm{~K}$ to $1.5 \mathrm{~K}$. The larger feature $\Delta$ remains unchanged. The temperature dependence $\mathrm{G}(\mathrm{T})$ measured at large carrier density can be fit with a $\log (\mathrm{T})$ dependence for $\mathrm{T} \leq 1 \mathrm{~K}$.

Device B2b differs from B2a by having a full gap, which reduces $G$ to zero at the CNP. Fig. 2k) shows $G_{d}$ as a function of $V_{g}$ and $V_{s d}$ at $230 \mathrm{mK}$. The line profile at the CNP is qualitatively similar to B2a, but $G_{d}$ is completely suppressed for small $V_{s d}$. Furthermore, the small gap feature $\delta$ is not readily visible but manifests only as a change in the slope of $G_{d}$ at $\left|V_{s d}\right|=1.5 \mathrm{mV}$. The larger feature $\Delta$ is shifted to higher voltages as well, with $\left|V_{s d}\right|=4 \mathrm{mV}$, and shows a larger overshoot before $G_{d}$ saturates at $2.3 \mathrm{e}^{2} / \mathrm{h}$. Tuning $V_{g}$ away from the CNP quenches the larger feature $\Delta$ but leaves the smaller $\delta$ intact in 
agreement to what is observed in B2a. In fig. 2d) the temperature dependence makes $\delta$ better visible. The small gap feature shrinks as $T$ is raised from $0.23 \mathrm{~K}$ to $1.5 \mathrm{~K}$, but still reduces $G_{d}$ to zero at $V_{s d}=0 \mathrm{mV}$. A further increase to $4 \mathrm{~K}$ completely suppresses $\delta$. The temperature change from $0.23 \mathrm{~K}$ to $1.5 \mathrm{~K}$ only reduces the overshoot in the larger feature $\Delta$. Increasing $T$ to $4 \mathrm{~K}$ yields a reduction of $\Delta$ and removes the overshoot.
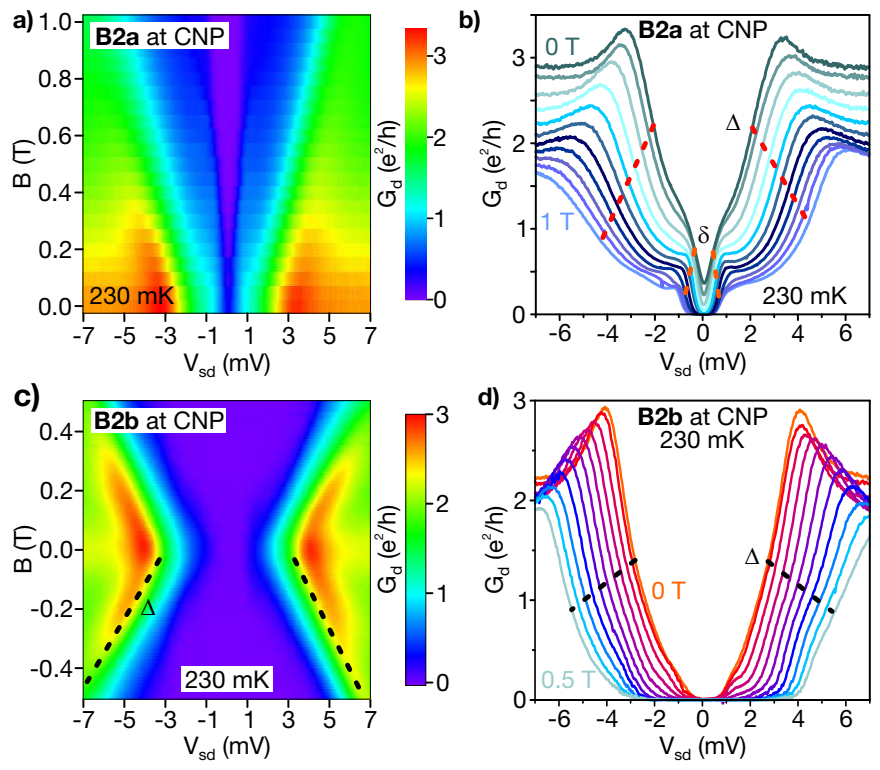

Figure 3: (a) Two-terminal conductance $G$ as a function of bias voltage $V_{s d}$ and perpendicular magnetic field $B$ of device $\mathrm{B} 2 \mathrm{a}$ at the $\mathrm{CNP}$ and $230 \mathrm{mK}$. The device is low-conducting at $B=0 \mathrm{~T}$ and smoothly evolves into an insulating state for $B>0.5 \mathrm{~T}$. (b) The corresponding line profiles. The dashed lines are guides to the eye for the linear scaling of $\Delta$ and $\delta$ with $B$. $\Delta$ scales as $2.8 \mathrm{meV} / \mathrm{T}$, whereas $\delta$ grows with $0.6 \mathrm{meV} / \mathrm{T}$. (c) $G$ as a function of $V_{s d}$ and $B$ of device B2b at the CNP and $230 \mathrm{mK}$. The device is insulating even at $B=0 \mathrm{~T}$. The gap $\Delta$ increases linearly with $B$. (d) The corresponding line profiles. The dashed line is a guide to the eye for the evolution of $\Delta$ with $6 \mathrm{meV} / \mathrm{T}$.

To find out about the origin of the two different features $\delta$ and $\Delta$ we perform differential conductance measurements $G_{d}\left(V_{s d}\right)$ as a function of perpendicular magnetic field, shown in figure 3. Both $\delta$ and $\Delta$ increase linearly with $|B|$ but with different slope. Taking the two inflection points of the $G_{d}\left(V_{s d}\right)$ traces as a measure for $\delta$ and $\Delta$, we can determine $\partial \delta / \partial B$ to $0.6 \mathrm{meV} / \mathrm{T}$, which is considerably larger than the Zeeman energy of a spin $1 / 2(0.12 \mathrm{meV} / \mathrm{T}$ for $\mathrm{g}=2)$. Looking at the large gap $\Delta$, we find $\partial \Delta / \partial B=2.8 \mathrm{meV} / \mathrm{T}$ for sample B2a (Fig. 3b) and $6 \mathrm{meV} / \mathrm{T}$ for sample B2b (Fig $3 \mathrm{~d}$ ). As sample B2b showed a full gap already at $B=0$, the magnetic field dependence of $\delta$ could not be determined here. Similarly, Velasco et al. observed $5.5 \mathrm{meV} / \mathrm{T}$ [20] for a fully gapped bilayer graphene device for a gap of similar size as $\Delta$.

The experiments clearly show two distinct gap-like features that occur in 
different ranges of charge carrier density and temperature, and that show a clearly different magnetic field dependence. We think that this observation can only be explained by the coexistence of different electronic phases next to each other in different parts of the samples. This conjecture is supported by the results of the current annealing procedures shown in figure 1, that can be understood by the existence of almost disorder-free, clean regions that are surrounded by more disordered areas 21, 22], where the current annealing has been less efficient. In the disordered regions the CNP lies outside the charge carrier densities that are accessible to us, due to chemical doping by adsorbates and residues. In this picture, sample B2a would be composed of a clean region close to contact B, and a more disordered rest of the sample. In sample B2b the clean region seems to be larger, occupying the center of the sample, but different CNP conductances between different terminals indicate that even in sample $\mathrm{B} 2 \mathrm{~b}$ there are regions with stronger disorder that are not evenly distributed, as illustrated in Fig. 4.

We assume that the clean phase is showing an interaction induced gap $\Delta$ close to the CNP with vanishing conductivity, and a distinct, BCS like shape. The large increase of $\Delta$ with $B$ of several meV per tesla has been predicted by theory for certain broken symmetry states like the QAH or QSH [12, 20].

a)

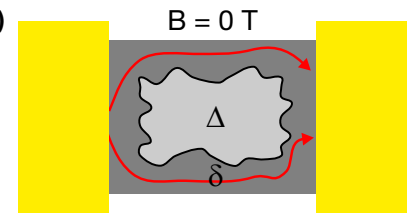

b)

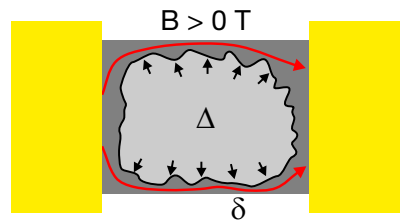

Figure 4: Sketch of the graphene device with the clean (light grey) and the disordered regions (dark grey) at the charge neutrality point. (a) In the absence of an external magnetic field, the clean region is gapped with $\Delta$ and the contacts do not couple to it. The disordered region remains conductive (red arrows), but experiences a transport gap $\delta$ due to localization. (b) In the presence of a perpendicular magnetic field the clean region expands, leading to a narrowing of the conducting areas $\delta$ and to a suppression of conductance.

The total conductance of sample B2a is composed of two parallel conduction channels, one through the clean region with gap $\Delta$ in the center of the sample, and another, more disordered one along the sample edges with gap-like feature $\delta$. The origin of the smaller feature $\delta$ is probably disorder induced localization 23 25]. For a disordered device such as a graphene ribbon with a predetermined geometry the application of a magnetic flux through the sample should lift the localization at least in part. In contrast to this scenario, the small gap in our experiment even increases when a perpendicular magnetic field is applied. We explain this by a change in geometry. The application of a perpendicular magnetic field increases the size of the inner clean region and thereby narrows down the conduction channel at the sample edge (Fig. 4b), leading to increased localization in the disordered part. The different response of the two conduction channels to magnetic field is reflected in the different magnetic field dependence of $\partial \delta / \partial B=0.6 \mathrm{meV} / \mathrm{T}$ and $\partial \Delta / \partial B=2.8 \mathrm{meV} / \mathrm{T}$. 
In sample B2b the initial distribution of the two phases is such that already at $\mathrm{B}=0$ the sample is mostly in the clean phase, and there is only a negligible conductance contribution of the disordered phase at the CNP. This is also the reason for the fact that only a large gap $\Delta$ can be observed in $G_{d}\left(V_{s d}\right)$, and that $\delta$ is feeble.

Because the metallic contacts will act as a heat sink during current annealing, we also have to assume that the disordered phase persists close to them. In fact, a narrow area of organic deposits can be clearly identified close to the metal contacts in scanning electron micrographs. This disordered region will then decouple the clean area in the sample center from the contacts and thus prevent edge states, that exist in the case of a QAH or QSH phase to couple to the metal contacts. Consequently, any phase in the center of the sample would show insulating behaviour in two terminal conductance, no matter if it has edge-states (QAH, QSH) or not (LAF). As we have shown the current annealing process to be inhomogeneous, we think that $\delta$ is a feature of the disordered graphene regions that persist at the edges of the device. However, as the size of the disordered region will vary from sample to sample, any distribution of $\delta$ versus $\Delta$-gaps may be realized. Different distributions of clean and disordered regions in the samples will lead to different $G_{d}\left(V_{s d}\right)$ traces around the CNP, with a full suppression of conductance at $V_{s d}=0$ when the clean region dominates, and some small, finite conductance when there is a considerable region of the disordered phase remaining. The application of a perpendicular magnetic field will always lead to a growth of the gapped $\Delta$-phase at the expense of the conducting $\delta$ phase, in this way opening a full gap in all samples at some finite value of B. We note that, although not discussed in detail, a small gap $\delta$ was also observed in [20]. It persists at high charge carrier densities and shows a small magnetic field dependence, in contrast to the stronger magnetic field scaling of $\Delta$ and in full agreement to our observations.

\section{Conclusions}

In conclusion, we measured non-linear conductance on multi-terminal, suspended bilayer graphene devices in a Hall-cross geometry as a function of charge carrier density and perpendicular magnetic field. We find that current annealing cleans the graphene inhomogeneously, which leads to samples that are composed of two different regions with distinct electronic properties. Both of these regions show gap-like features at the CNP, as visible in differential conductance $G_{d}\left(V_{s d}\right)$. We extract two gaps of different magnitude that show a different temperature dependence and respond differently to magnetic field. Attributing the two gaps to clean and disordered sample regions, we find that the initial distribution of the two regions differs from sample to sample, but that the overall density and magnetic field dependence of the conductance of these two regions is similar. We argue that one of the two regions is a conductive, disordered phase localized at the sample edges that shows a zero bias anomaly over a large range in charge carrier density due to disorder induced localization, and a clean phase in the center of the sample that shows an interaction induced gap close to the 
charge neutrality point with strongly suppressed conductance. The application of a perpendicular magnetic field favors the expansion of the clean phase in the sample center and shrinks the disordered conducting areas, and therefore pronounces the signature of the interaction effects in differential conductance.

\section{Acknowledgments}

This work was financed by the Swiss NSF, the ESF programme Eurographene, the EU FP7 project $\mathrm{SE}^{2} \mathrm{ND}$, the Swiss NCCR Nano and QSIT. We are grateful to M. Kharitonov and C. N. Lau for discussions.

\section{References}

[1] K. S. Novoselov, A. K. Geim, S. V. Mozorov, D. Jiang, Y. Zhang, S. V. Dubonos, I. V. Grigorieva, A. A. Firsov, Science 306 (2004) 666.

[2] S. Das Sarma, S. Adam, E. H. Hwang, E. Rossi, Rev. Mod. Phys. 83 (2011) 407.

[3] K. Bolotin, K. Sikes, Z. Jiang, M. Klima, G. Fudenberg, J. Hone, P. Kim, H. Stormer, Solid State Commun. 146 (2008) 351.

[4] B. E. Feldman, J. Martin, A. Yacoby, Nature Phys. 5 (2009) 889.

[5] E. McCann, V. I. Fal'ko, Phys. Rev. Lett. 96 (2006) 086805.

[6] J. Nilsson, A. H. Castro-Neto, N. M. R. Peres, F. Guinea, Phys. Rev. B 73 (2006) 214418.

[7] M. Ezawa, Physica E 40 (2007) 269.

[8] X.-F. Wang, T. Chakraborty, Phys. Rev. B 75 (2007) 041404.

[9] Y. Barlas, R. Côtè, K. Nomura, A. H. MacDonald, Phys. Rev. Lett. 101 (2008) 097601.

[10] H. Min, G. Borghi, M. Polini, A. H. MacDonald, Phys. Rev. B 77 (2008) 041407.

[11] R. Nandkishore, L. Levitov, Phys. Rev. B 82 (2010) 115124.

[12] F. Zhang, J. Jung, G. A. Fiete, Q. Niu, A. H. MacDonald, Phys. Rev. Lett. 106 (2011) 156801.

[13] E. Rossi, S. Das Sarma, Phys. Rev. Lett. 107 (2011) 155502.

[14] M. Kharitonov, arXiv:1109.1553v2.

[15] Y. Lemonik, I. Aleiner, V. I. Fal'ko, Phys. Rev. B 85 (2012) 245451.

[16] O. Vafek, K. Yang, Phys. Rev. B 81 (2010) 041401. 
[17] Y. Lemonik, I. L. Aleiner, C. Toke, V. I. Fal'ko, Phys. Rev. B 82 (2010) 201408.

[18] M. Kharitonov, Phys. Rev. Lett. 109 (2012) 046803.

[19] F. Freitag, J. Trbovic, M. Weiss, C. Schöneneberger, Phys. Rev. Lett. 108 (2012) 076602.

[20] J. Velasco, Jr., L. Jing, W. Bao, Y. Lee, P. Kratz, V. Aji, M. Bockrath, C. N. Lau, C. Varma, R. Stillwell, D. Smirnov, F. Zhang, J. Jung, A. H. MacDonald, Nat. Nanotechnol. 7 (2012) 156.

[21] S. Das Sarma, E. H. Hwang, E. Rossi, Phys. Rev. B 81 (2010) 161407.

[22] E. H. Hwang, S. Das Sarma, Phys. Rev. B 82 (2010) 081409.

[23] R. V. Gorbachev, F. V. Tikhonenko, A. S. Mayorov, D. W. Horsell, A. K. Savchenko, Phys. Rev. Lett. 98 (2007) 176805.

[24] J. B. Oostinga, B. Sacépé, M. F. Craciun, A. F. Morpurgo, Phys. Rev. B 81 (2010) 193408.

[25] J.-M. Poumirol, A. Cresti, S. Roche, W. Escoffier, M. Goiran, X. Wang, X. Li, H. Dai, B. Raquet, Phys. Rev. B 82 (2010) 041413. 